\title{
Hyperfine splitting in a realistic basis for baryons
}

\author{
R. Sartor and Fl. Stancu \\ Université de Liège, Institut de Physique B5, Sart Tilman, B-4000 Liège 1, Belgium
}

(Received 12 June 1984)

\begin{abstract}
We calculate the positive- and negative-parity spectrum of baryons by diagonalizing the hyperfine Hamiltonian in a space corresponding to the SU(6) multiplets $\underline{56}, \underline{70}$, and $\underline{20}$. The highest spin considered is $J=\frac{7}{2}$ for positive parity and $J=\frac{5}{2}$ for negative parity. The main difference with the work of Isgur and Karl based on the harmonic oscillator is that we consider the more realistic unperturbed wave functions derived variationally by Carlson, Kogut, and Pandharipande in a flux-tube quark model. The finite size of the quark is also taken into account.
\end{abstract}

\section{INTRODUCTION}

Recently Carlson, Kogut, and Pandharipande ${ }^{1}$ (henceforth abbreviated as CKP) have provided variational wave functions for describing the nonstrange mesons and baryons. They consider a semirelativistic Hamiltonian with relativistic kinetic energy for the quarks and an adiabatic potential derived from a flux-tube quark model. In the case of a $q \bar{q}$ pair the potential is a linear combination of a Coulomb and a linear confinement term. For a color-singlet $q q q$ system they have shown that the potential energy can be expressed as a sum of three pair potentials and a three-body potential which depends on the tension in the flux tubes. Accordingly, a three-body correlation factor has been included in the baryonic wave functions. Both the two- and three-body parts are parametrized functions of the relative position of the quarks and the parameters are found by minimizing the energy.

The present study is intended as a complement to the work of Ref. 1. We analyze the role of the hyperfine interaction $^{2}$ starting from a nonperturbed SU(6) basis formed with the variational wave functions of Ref. 1. We calculate the nonstrange-baryon spectra closely following the method applied by Isgur and $\mathrm{Karl}^{3-5}$ for a harmonic confinement. Apart from the more realistic long-distance behavior of the confining potential, another important advantage in using the CKP wave functions is that one has a consistent approach both for the color-electric and -magnetic interactions. The Hamiltonian of Ref. 1, yields a unique unperturbed spectrum which does not have to be adjusted separately for each parity. Moreover, there is no degeneracy to be removed as in the harmonic-oscillator case. Therefore such a procedure has more predictive power.

The unperturbed space considered in this analysis is spanned by the $\underline{56}\left(0^{+}, 2^{+}\right), \underline{56^{\prime}}\left(0^{+}\right), \underline{70}\left(0^{+}, 1^{-}, 2^{+}\right)$, and $20\left(1^{+}\right) \mathrm{SU}(6)$ multiplets. The outcome of our calculations is the spectrum of $N$ and $\Delta$ and the mixing angles for positive-parity states up to $J=\frac{7}{2}$ and negative-parity states up to $J=\frac{5}{2}$.

More recently, Carlson, Kogut, and Pandharipande have considered other variational wave functions ${ }^{6}$ which in a $q q q$ system include pair correlations due to the spinspin color-magnetic interaction. In the excited spectrum of baryons they consider only the $1^{-}$state and the $0^{+}$ breathing mode and calculate energy levels up to $J=\frac{3}{2}$ for positive parity and $J=\frac{5}{2}$ for negative parity.

In the next section we summarize the ingredients of our calculations. In Sec. III we present the baryon spectrum obtained from the diagonalization of the hyperfine interaction modified to include finite-size effects and for comparable $J$ values we discuss them in the light of Ref. 6. Concluding remarks are given in Sec. IV. Appendix A gives a detailed analysis of the normalization constants of the CKP wave functions. In Appendix B we show that the 66 matrix elements of the spin-spin and tensor parts of the hyperfine interaction can be reduced to linear combinations of 13 three-dimensional integrals when the finite size of the quark is taken into account. Therefore the integrals can be computed with a better precision than usually allowed by the Monte Carlo method. ${ }^{1,6}$

\section{THE BASIS}

In our calculations of the baryon spectra the space part of the wave functions is treated as in Ref. 1. Accordingly the wave function of a $q q q$ system is chosen to be of the form

$$
\psi_{n}\left(\overrightarrow{\mathrm{r}}_{12}, \overrightarrow{\mathrm{r}}_{13}, \overrightarrow{\mathrm{r}}_{23}\right)=F_{123} \prod_{i<j} f\left(r_{i j}\right) \Phi_{n}\left(\overrightarrow{\mathrm{r}}_{12}, \overrightarrow{\mathrm{r}}_{13}, \overrightarrow{\mathrm{r}}_{23}\right),
$$

where $f$ and $F_{123}$ are the two- and three-body parts of the ground-state wave function $n=0$. The functions $\Phi_{n}$ with $n \neq 0$ are defined in Eqs. (2.8) to (2.17). The function $f$ is parametrized as

$$
\begin{aligned}
& \ln f\left(r_{i j}\right)=-W\left(r_{i j}\right) \lambda_{1} r_{i j}-\left[1-W\left(r_{i j}\right)\right] \lambda_{1.5} r_{i j}{ }^{1.5}, \\
& W\left(r_{i j}\right)=\frac{1+\exp \left(-r_{0} / a\right)}{1+\exp \left[\left(r_{i j}-r_{0}\right) / a\right]},
\end{aligned}
$$

where $r_{i j}$ is the distance between the quarks $i$ and $j$ $(i, j=1,2,3)$. One can see that the function $f\left(r_{i j}\right)$ decreases at large $r_{i j}$, as implied by the linear confinement. 
The three-body part is chosen as

$$
F_{123}=1-\beta \sqrt{\sigma}\left[\sum_{i} r_{i 4}-\frac{1}{2} \sum_{i<j} r_{i j}\right],
$$

where $r_{i 4}$ is the distance between the quark $i$ and a point $\vec{r}_{4}$, where the flux tubes meet at $120^{\circ}$. For angles larger than $120^{\circ}$ this point becomes identical to one of the quark positions $r_{i}(i=1,2,3)$, as explained in Ref. 1 .

The quantities $\lambda_{1}, \lambda_{1.5}, r_{0}, a$, and $\beta$ are the variational parameters found by CKP:

$$
\begin{aligned}
& \lambda_{1}=0.637 \mathrm{fm}^{-1}, \\
& \lambda_{1.5}=1.40 \mathrm{fm}^{-1.5}, \\
& r_{0}=0.12 \mathrm{fm}, \\
& a=0.12 \mathrm{fm}, \\
& \beta=0.25 \mathrm{GeV}^{-1},
\end{aligned}
$$

and $\sqrt{\sigma}$ takes the conventional value of the string-tension constant

$$
\sqrt{\sigma}=1 \mathrm{GeV} / \mathrm{fm} \text {. }
$$

As usual, we express the relative coordinates $r_{i j}$ in terms of the Jacobi relative coordinates

$$
\begin{aligned}
& \vec{\rho}=\frac{1}{\sqrt{2}}\left(\overrightarrow{\mathrm{r}}_{1}-\overrightarrow{\mathrm{r}}_{2}\right), \\
& \vec{\lambda}=\frac{1}{\sqrt{6}}\left(\overrightarrow{\mathrm{r}}_{1}+\overrightarrow{\mathrm{r}}_{2}-2 \overrightarrow{\mathrm{r}}_{3}\right) .
\end{aligned}
$$

By using the notations of Isgur and $\mathrm{Karl}^{4,5}$ for angular momenta $L=0,1,2$ and projection $M=0$ the normalized $\psi_{n}$ considered in this study can be written as

$$
\begin{aligned}
& \psi_{00}^{S}=N_{00}^{S} F, \\
& \psi_{00}^{S^{\prime}}=N_{00}^{S^{\prime}}\left[1-\alpha\left(\rho^{2}+\lambda^{2}\right)\right] F, \\
& \psi_{00}^{\rho}=N_{00}^{\rho} \vec{\rho} \cdot \overrightarrow{\lambda F}, \\
& \psi_{00}^{\lambda}=N_{00}^{\rho} \frac{1}{2}\left(\rho^{2}-\lambda^{2}\right) F \\
& \psi_{10}^{\rho}=N_{10}^{\rho} \rho_{0} F \\
& \psi_{10}^{\lambda}=N_{10}^{\rho} \lambda_{0} F \\
& \psi_{10}^{A}=N_{10}^{A}\left(\rho_{-} \lambda_{+}-\rho_{+} \lambda_{-}\right) F, \\
& \psi_{20}^{S}=N_{20}^{S}\left[3\left(\rho_{0}^{2}+\lambda_{0}^{2}\right)-\left(\rho^{2}+\lambda^{2}\right)\right] F, \\
& \psi_{20}^{\rho}=N_{20}^{\rho}\left(3 \rho_{0} \lambda_{0}-\vec{\rho}^{2} \cdot \vec{\lambda}\right) F, \\
& \psi_{20}^{\lambda}=N_{20}^{\rho} \frac{1}{2}\left[3\left(\rho_{0}{ }^{2}-\lambda_{0}{ }^{2}\right)-\left(\rho^{2}-\lambda^{2}\right)\right] F,
\end{aligned}
$$

where

$$
\rho_{ \pm}=\rho_{x} \pm i \rho_{y}, \quad \lambda_{ \pm}=\lambda_{x} \pm i \lambda_{y},
$$

and

$$
F=F_{123} \prod_{i<j} f\left(r_{i j}\right) .
$$

Let us remark that the orthogonality of the above states has been ensured by a proper choice of $\Phi_{n}$ in Eq. (2.1). In Appendix A we show that the constant $\alpha$ and the 8 nor- malization factors $N_{L M}^{\mathrm{sym}}$, where the superscript "sym" designates the permutation symmetry, can be expressed in terms of five independent three-dimensional integrals. The three integration variables are $\rho, \lambda$, and $x=\vec{\rho} \cdot \vec{\lambda} / \rho \lambda$. The value obtained for $\alpha$ from the orthogonality between (2.8) and (2.9) is

$$
\alpha=2.53 \mathrm{fm}^{-2} \text {. }
$$

The hyperfine interaction ${ }^{2}$ is modified to include the finite size $\Lambda$ of the quark as in Ref. 6. Then for the quark pair 12 the spin-spin and tensor interactions read

$$
\begin{aligned}
V_{\mathrm{SS}}= & \frac{4 \sqrt{2} \pi \alpha_{S}}{9 m^{2}} \frac{1}{\left(2 \pi \Lambda^{2}\right)^{3 / 2}} e^{-\rho^{2} / 2 \Lambda^{2} \overrightarrow{\mathrm{S}}_{1} \cdot \overrightarrow{\mathrm{S}}_{2}} \\
V_{\mathrm{T}}= & \frac{\alpha_{S}}{\sqrt{2} m^{2} \rho^{3}}\left[\operatorname{erf}\left[\frac{\rho}{\sqrt{2} \Lambda}\right]\right. \\
& \left.\quad-\frac{\sqrt{2}}{3 \sqrt{\pi}} \frac{\rho^{3}}{\Lambda^{3}}\left[1+3 \frac{\Lambda^{2}}{\rho^{2}}\right] e^{-\rho^{2} / 2 \Lambda^{2}}\right] \\
\times & {\left[\frac{1}{\rho^{2}} \overrightarrow{\mathrm{S}}_{1} \cdot \vec{\rho} \overrightarrow{\mathrm{S}}_{2} \cdot \vec{\rho}-\frac{1}{3} \overrightarrow{\mathrm{S}}_{1} \cdot \overrightarrow{\mathrm{S}}_{2}\right] }
\end{aligned}
$$

As we consider only nonstrange baryons we deal with completely symmetric states in flavor, spin, and space. Then the contribution of all three pairs is given by three times the contribution of the pair 12 in each given state. The value of the strong-interaction coupling constant $\alpha_{S}$ is taken as in Ref. 1, i.e.,

$$
\frac{4}{3} \frac{\alpha_{S}}{\hbar c}=0.5 \text {. }
$$

The quantity $\Lambda$ is assumed to be a free parameter. This will be discussed in the next section.

We combine the spatial wave functions of Eqs. (2.8)-(2.17) with the quark spin and flavor in order to construct totally symmetric wave functions. To describe them in a SU(6) basis we follow the notations of Isgur and $\mathrm{Karl}{ }^{3-5}$ i.e., each state is represented by the normalized ket $\left|{ }^{2 S+1} X\left(\mu, L^{\pi}\right) J^{\pi}\right\rangle$, where $X=N$ or $\Delta, S, L$, and $J$ are the spin, orbital, and total angular momenta, $\pi$ is the parity, and $\mu$ is the SU(6) multiplet.

In the following section we present the results obtained by diagonalizing the hyperfine interaction $V_{\mathrm{SS}}+V_{\mathrm{T}}$ in the above-mentioned basis.

\section{RESULTS AND DISCUSSION}

The results obtained by the diagonalization of the Hamiltonian containing the hyperfine interaction in the basis described in Sec. II are given in Tables I and II for $\pi=+1$ and $\pi=-1$ states, respectively. These results have been obtained with a precision of $0.5 \%$ in the computation of the integrals $S_{i}(i=1-7)$ and $T_{i}(i=1-6)$ entering the matrix elements of the spin-spin and tensor interactions (see Appendix B).

To allow a comparison with Ref. 6, we first consider the same values for the quark mass, the finite-size constant, and the energy constant, namely, $m=360 \mathrm{MeV}$, $\Lambda=0.13 \mathrm{fm}$, and $E_{0}^{B}=-1265 \mathrm{MeV}$. Later on, we shall let $m$ and $\Lambda$ vary. A comment is in order for the varia- 
TABLE I. Nonstrange baryons of positive parity. (1) Mass spectrum (this work). (2) Mixing angles (this work). (3) Mass spectrum (Ref. 6). (4) Experimental mass spectrum (Ref. 7). Mass spectra are in MeV. Columns (1), (2), and (3) have been obtained with $m=360 \mathrm{MeV}$ and $\Lambda=0.130 \mathrm{fm}$.

\begin{tabular}{|c|c|c|c|c|c|c|c|c|}
\hline State & (1) & & & (2) & & & (3) & (4) \\
\hline${ }^{4} N\left(\underline{70}, 2^{+}\right) \frac{7}{2}^{+}$ & 2028 & (1) & & & & & & $1950-2050$ \\
\hline${ }^{4} \Delta\left(\underline{56}, 2^{+}\right) \frac{7}{2}^{+}$ & 1985 & (1) & & & & & & $1910-1960$ \\
\hline${ }^{2} N\left(\underline{56}, 2^{+}\right) \frac{5}{2}^{+}$ & 1860 & 0.864 & -0.504 & $7 \times 10^{-3}$ & & & & $1670-1690$ \\
\hline${ }^{2} N\left(\underline{70}, 2^{+}\right) \frac{5}{2}^{+}$ & 2006 & -0.499 & -0.858 & -0.120 & & & & \\
\hline${ }^{4} N\left(\underline{70}, 2^{+}\right) \frac{5}{2}^{+}$ & 2061 & -0.066 & -0.100 & 0.993 & & & & \\
\hline${ }^{4} \Delta\left(\underline{56}, 2^{+}\right) \frac{5}{2}^{+}$ & 1997 & 0.599 & 0.800 & & & & & $1890-1920$ \\
\hline${ }^{2} \Delta\left(70,2^{+}\right) \frac{5}{2}^{+}$ & 2011 & 0.800 & -0.599 & & & & & \\
\hline${ }^{4} N\left(\underline{70}, 0^{+}\right) \frac{3}{2}^{+}$ & 1859 & 0.067 & -0.859 & 0.507 & 0.007 & $7 \times 10^{-4}$ & & $1690-1800$ \\
\hline${ }^{2} N\left(\underline{56}, 2^{+}\right) \frac{3}{2}+$ & 1956 & -0.907 & -0.195 & -0.215 & 0.299 & 0.058 & & \\
\hline${ }^{2} N\left(\underline{70}, 2^{+}\right) \frac{3}{2}^{+}$ & 2008 & -0.350 & 0.444 & 0.802 & -0.182 & -0.069 & & \\
\hline${ }^{4} N\left(\underline{70}, 2^{+}\right) \frac{3}{2}^{+}$ & 2033 & -0.205 & -0.155 & -0.225 & -0.780 & -0.524 & & \\
\hline${ }^{2} N\left(\underline{20}, 1^{+}\right) \frac{3}{2}^{+}$ & 2077 & -0.093 & -0.046 & -0.060 & -0.518 & 0.847 & & \\
\hline${ }^{4} \Delta\left(\underline{56}, 0^{+}\right) \frac{3}{2}^{+}$ & 1308 & 0.991 & -0.116 & -0.053 & 0.035 & & 1240 & $1230-1234$ \\
\hline${ }^{4} \Delta\left(\underline{56}^{\prime}, 0^{+}\right) \frac{3}{2}^{+}$ & 1893 & 0.113 & 0.993 & -0.031 & 0.030 & & 1847 & $1500-1900$ \\
\hline${ }^{4} \Delta\left(\underline{56}, 2^{+}\right) \frac{3}{2}^{+}$ & 1986 & 0.068 & 0.035 & 0.842 & -0.534 & & & $1860-2160$ \\
\hline${ }^{2} \Delta\left(\underline{70}, 2^{+}\right) \frac{3}{2}^{+}$ & 2010 & 0.002 & 0.009 & -0.536 & -0.844 & & & \\
\hline${ }^{2} N\left(\underline{56}, 0^{+}\right) \frac{1}{2}^{+}$ & 1092 & -0.988 & -0.106 & 0.110 & 0.024 & $-7 \times 10^{-4}$ & 940 & 940 \\
\hline${ }^{2} N\left(\underline{56}^{\prime}, 0^{+}\right) \frac{1}{2}^{+}$ & 1712 & 0.093 & -0.988 & -0.121 & 0.001 & $-10^{-4}$ & 1583 & $1400-1480$ \\
\hline${ }^{2} N\left(\underline{70}, 0^{+}\right) \frac{1}{2}^{+}$ & 1868 & 0.116 & -0.108 & 0.973 & -0.166 & 0.025 & & $1680-1740$ \\
\hline${ }^{4} N\left(\underline{70}, 2^{+}\right) \frac{1}{2}^{+}$ & 1996 & 0.041 & -0.014 & 0.158 & 0.904 & -0.395 & & \\
\hline${ }^{2} N\left(\underline{20}, 1^{+}\right) \frac{1}{2}^{+}$ & 2076 & -0.014 & 0.003 & -0.041 & -0.394 & -0.918 & & \\
\hline${ }^{2} \Delta\left(\underline{70}, 0^{+}\right) \frac{1}{2}^{+}$ & 1934 & 0.99985 & 0.01758 & & & & & $1850-1950$ \\
\hline${ }^{4} \Delta\left(\underline{56}, 2^{+}\right) \frac{1}{2}^{+}$ & 1970 & -0.01758 & 0.99985 & & & & & \\
\hline
\end{tabular}

tion of $m$. The unperturbed spectrum used in these calculations is taken from Ref. 1 and corresponds to the mass $m_{0}=313 \mathrm{MeV}$. In cases where we use a different quark mass, we calculate the effect of $\Delta m=m-m_{0}$ by the perturbation theory.
In making the comparison, one should have in mind that the essential difference between the wave functions used here and those of Ref. 6 is that there the wave functions contain short-range spin-spin correlations. In our work we include mixing between different spin and angu-

TABLE II. Same as Table I for nonstrange baryons of negative parity.

\begin{tabular}{|c|c|c|c|c|c|}
\hline State & (1) & & & (3) & (4) \\
\hline${ }^{4} N\left(70,1^{-}\right) \frac{5}{2}^{-}$ & 1690 & (1) & & 1661 & $1660-1690$ \\
\hline${ }^{2} N\left(\underline{70}, 1^{-}\right) \frac{3}{2}^{-}$ & 1579 & 0.998 & 0.069 & 1446 & $1510-1530$ \\
\hline${ }^{4} N\left(\underline{70}, 1^{-}\right) \frac{3}{2}^{-}$ & 1726 & -0.069 & 0.998 & 1628 & $1670-1730$ \\
\hline${ }^{2} \Delta\left(\underline{70}, 1^{-}\right) \frac{3}{2}^{-}$ & 1657 & (1) & & 1564 & $1630-1740$ \\
\hline${ }^{2} N\left(\underline{70}, 1^{-}\right) \frac{1}{2}^{-}$ & 1568 & 0.945 & -0.327 & 1399 & $1520-1560$ \\
\hline${ }^{4} N\left(70,1^{-}\right) \frac{1}{2}^{-}$ & 1673 & 0.327 & 0.945 & 1514 & $1620-1680$ \\
\hline${ }^{2} \Delta\left(\underline{70}, 1^{-}\right) \frac{1}{2}^{-}$ & 1657 & (1) & & 1566 & $1600-1650$ \\
\hline
\end{tabular}




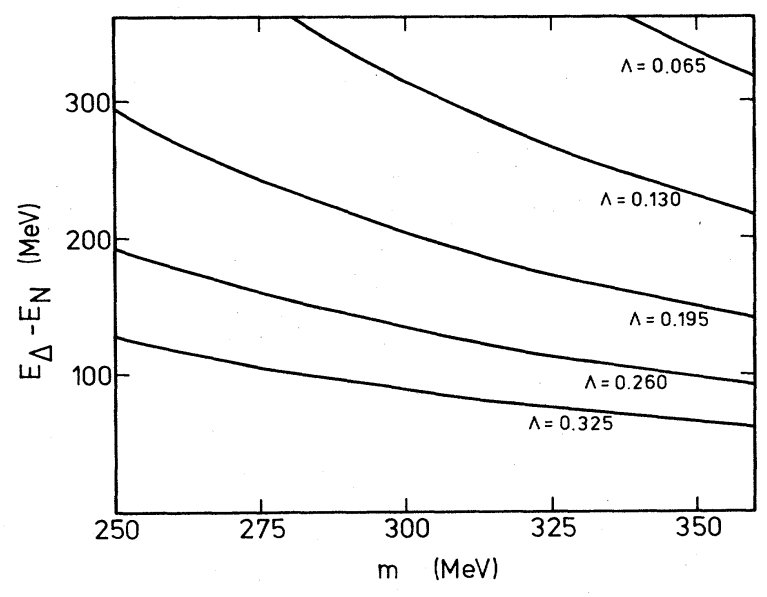

FIG. 1. The quark-mass dependence of the $\Delta-N$ splitting for different values of the finite-size parameter $\Lambda$ (in $\mathrm{fm}$ ). The experimental value lies in the range (290-294) $\mathrm{MeV}$.

lar momenta, but the correlations introduced in this way are not short range.

As a general trend the calculated positive parity levels are situated within the experimental error bars except for those dominated by $L=0$ and/or $S=\frac{1}{2}$ configurations. Such a result is consistent with the conclusion reached in Ref. 6 according to which extra spin-spin correlations have to be added to the wave functions containing quark pairs with $L=0, S=0$. Comparing columns (1) and (3) of Table I one can see that these correlations help to bring down the ground and first excited states of $N$ and $\Delta$ by $\sim 150 \mathrm{MeV}$ and $\sim 50 \mathrm{MeV}$, respectively.

For negative-parity states the situation is different. Table II shows that the energies and also the mixing angles calculated with the present approach are in better agreement with experiment ${ }^{8}$ than those found in Ref. 6 . This suggests that for the Hamiltonian which has been used above and in Ref. 6, our negative-parity wave functions have more appropriate spin-spin correlations for calculating mixing angles.

To our knowledge there are no mixing angles extracted

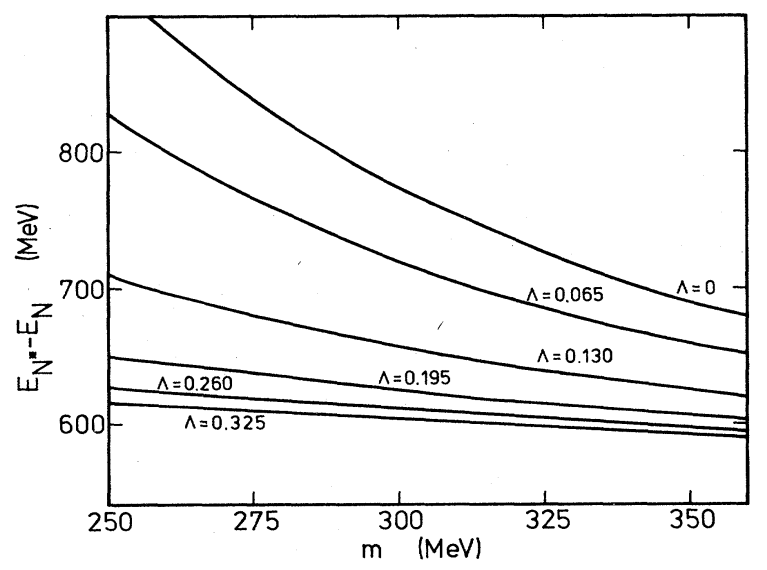

FIG. 2. Same as Fig. 1 for the $N^{*}-N$ splitting. The experimental value lies in the range $(460-540) \mathrm{MeV}$.

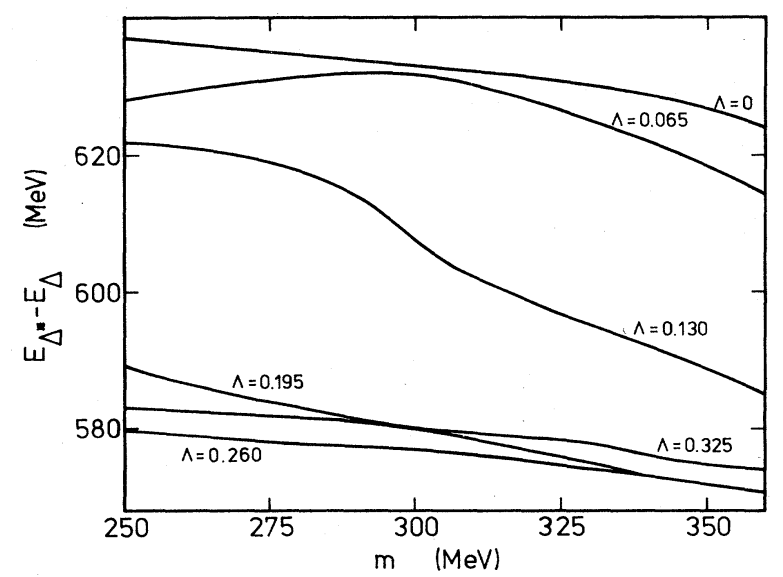

FIG. 3. Same as Fig. 1 for the $\Delta^{*}-\Delta$ splitting. The experimental value lies in the range (266-670) $\mathrm{MeV}$.

from experimental data for $\pi=+1$ levels. Table I shows cases of strong mixture for practically all $J$ values. This had to be expected from the closeness of the unperturbed levels $\underline{56}\left(0^{+}\right), \underline{70}\left(0^{+}\right), \underline{56}\left(2^{+}\right), \underline{70}\left(2^{+}\right)$, and $\underline{20}\left(1^{+}\right)$. Note, in particular, that the hyperfine coupling has inverted the weight of the $\underline{56}\left(2^{+}\right)$with that of the $\underline{70}\left(0^{+}\right)$and $\underline{70}\left(2^{+}\right)$ configurations in the $\Delta\left(\frac{5}{2}^{+}\right)$and $N\left(\frac{3}{2}^{+}\right)$spectra, respectively. A similar result has been also obtained by Isgur and $\mathrm{Karl}^{4}{ }^{4}$

In Ref. 6, $\Lambda$ has been chosen to reproduce the $\pi$ - $\rho$ or equivalently the $N-\Delta$ splitting. One may ask how the above results depend on this choice. We have considered other values of $m$ as well as of $\Lambda$ because they both affect the hyperfine splitting. The mass has been allowed to vary in the interval $250-360 \mathrm{MeV}$ since values down to $230 \pm 15 \mathrm{MeV}$ of the quark constituent mass are consistent with chiral-symmetry breaking and QCD sum rules. ${ }^{9}$ For $\Lambda$ we have taken values in the range $0.0-0.325 \mathrm{fm}$ with a step $0.065 \mathrm{fm}$, i.e., half the value of $\Lambda$ chosen in Ref. 6 . A change in $\Lambda$ modifies the effects of the hyperfine interaction at short separation distances. In calculating the spectrum such a change can to some extent simulate the effect of short-range correlations. For the various $\Lambda$ considered, we show in Figs. $1-3$ the results for the $\Delta-N, N^{*}-N$, and $\Delta^{*}-\Delta$ splittings as a function of $m$. Except for $\Delta^{*}-\Delta$ splitting (note the range on the ordinate scale) there is a pronounced dependence on the quark mass which, however, becomes weaker as $\Lambda$ increases. From Fig. 1 one can see that the present model can accommodate the whole considered mass range if $\Lambda$ is properly chosen. For instance, with $m=250$ and $\Lambda=0.195 \mathrm{fm}$ we can obtain the same kind of fit as in Ref. 6. This is indicated in Table III.

TABLE III. $\Delta-N, N^{*}-N$, and $\Delta^{*}-\Delta$ splittings in MeV. (1) This work with $m=250 \mathrm{MeV}$ and $\Lambda=0.195 \mathrm{fm}$. (2) Reference $6(m=360 \mathrm{MeV}$ and $\Lambda=0.130 \mathrm{fm})$. (3) Experiment (Ref. 7).

\begin{tabular}{rccc}
\hline \hline & $(1)$ & $(2)$ & $(3)$ \\
\hline$\Delta-N$ & 294 & 300 & $290-294$ \\
$N^{*}-N$ & 651 & 643 & $460-540$ \\
$\Delta^{*}-\Delta$ & 589 & 607 & $266-670$ \\
\hline \hline
\end{tabular}


From Fig. 2 it appears that values even larger than $\Lambda=0.195 \mathrm{fm}$ would be favored by the $N^{*}-N$ splitting.

\section{SUMMARY}

In this work we have calculated the nonstrange-baryon spectra up to $J^{\pi}=\frac{7}{2}^{+}$and $J^{\pi}=\frac{5}{2}^{-}$by diagonalizing the hyperfine interaction-modified for finite-size effects-in a basis obtained from the variational wave functions of Ref. 1 and which spans the $\underline{56}, \underline{70}$, and $\underline{20}$ SU(6) multiplets.

We have analyzed the dependence of the spectrum on the quark mass $m$ and the size parameter $\Lambda$ which both influence the coupling of various states. Our results suggest that the present approach leaves an ambiguity in the choice of $m$ and $\Lambda$. There are several $(m, \Lambda)$ sets which give results very similar to those of Ref. 6 . As the Roper resonance has too high an energy for all choices of parameters, it seems desirable to improve the first $0^{+}$breathing mode. The variational wave functions with spin-spin correlations ${ }^{6}$ give lower energies and therefore more accurate values ${ }^{10}$ than ours for the chosen Hamiltonian. In spite of this, our wave functions give better mixing angles for the negative-parity states and the diagonalization method being simpler allows more extensive calculations. It would be useful to make a comparison with results given by variational wave functions containing spin-spin correlations for $J^{\pi}>\frac{3}{2}^{+}$levels and to further test the configuration mixings by calculating decay amplitudes. ${ }^{11}$

\section{ACKNOWLEDGMENTS}

We thank H. Caprasse, G. Karl, and J. Paton for fruitful discussions and are especially grateful to D. M. Brink for a thorough and critical reading of the manuscript.

\section{APPENDIX A}

In this and the following appendix, we use the compact notation

$$
\langle F|(\cdots)| F\rangle=\int d^{3} \rho d^{3} \lambda(\cdots) F^{2}(\vec{\rho}, \vec{\lambda}),
$$

where $(\cdots)$ stands for any expression.

By taking into account that $F^{2}(\vec{\rho}, \vec{\lambda})$ depends only on $\rho$, $\lambda$, and $\vec{\rho} \cdot \vec{\lambda}$, one can show easily that the normalization factors appearing in Eqs. (2.8) to (2.12) can be written as

$$
\begin{aligned}
& \left(N_{00}^{s}\right)^{-2}=N_{1} \\
& \left(N_{00}^{s^{\prime}}\right)^{-2}=\frac{1}{4}\left[\frac{N_{1}}{N_{2}}\right)^{2}\left(N_{3}+2 N_{4}+N_{5}\right)-N_{1}, \\
& \left(N_{00}^{\rho}\right)^{-2}=\frac{1}{4}\left(N_{3}-2 N_{4}+N_{5}\right) \\
& \left(N_{20}^{s}\right)^{-2}=\frac{7}{5}\left(N_{3}+N_{5}\right)-2 N_{4}, \\
& \left(N_{20}^{\rho}\right)^{-2}=\frac{1}{20}\left(N_{3}+N_{5}\right)+\frac{1}{2} N_{4} \\
& \left(N_{10}^{A}\right)^{-2}=2 N_{4}-\frac{1}{3}\left(N_{3}+N_{5}\right) \\
& \left(N_{10}^{\rho}\right)^{-2}=\frac{1}{3} N_{2},
\end{aligned}
$$

where $N_{1} \ldots N_{5}$ are defined by

$$
\begin{aligned}
& N_{1}=\langle F \mid F\rangle, \\
& N_{2}=\left\langle F\left|\rho^{2}\right| F\right\rangle, \\
& N_{3}=\left\langle F\left|\rho^{4}\right| F\right\rangle, \\
& N_{4}=\left\langle F\left|\rho^{2} \lambda^{2}\right| F\right\rangle, \\
& N_{5}=\left\langle F\left|\lambda^{4}\right| F\right\rangle .
\end{aligned}
$$

Note that the six-dimensional integrals (A9)-(A13) reduce to three-dimensional ones, three out of the six integrations involved in the calculation of the $N_{i}$ 's being trivial. The remaining variables are $\rho, \lambda$ and $x=\vec{\rho} \cdot \vec{\lambda} / \rho \lambda$ and the integrals can be computed without using the Monte Carlo method. The values we obtained are given in Table IV.

In the same manner, one can show that the constant $\alpha$ which appears in Eq. (2.9) can be written as

$$
\alpha=\frac{1}{2} \frac{N_{1}}{N_{2}} .
$$

In deriving (A14) and reducing all normalization constants to the linear combinations (A2)-(A18), use has been made of the properties

$$
\begin{aligned}
\left\langle F\left|\rho^{2}\right| F\right\rangle=\left\langle F\left|\lambda^{2}\right| F\right\rangle, & \\
\left\langle F\left|\rho^{2} \lambda^{2} P_{2}(x)\right| F\right\rangle= & \frac{3}{8}\left\langle F\left|\left(\rho^{4}+\lambda^{4}\right)\right| F\right\rangle \\
& -\frac{5}{4}\left\langle F\left|\rho^{2} \lambda^{2}\right| F\right\rangle .
\end{aligned}
$$

These relations can be easily proved by taking into account that $F$ is invariant under all permutations of three particles.

\section{APPENDIX B}

We present here the nonvanishing matrix elements of the spin-spin [Eq. (2.14)] and tensor [Eq. (2.20)] parts of the three-quark Hamiltonian in units of $C_{\mathrm{SS}}$ and $C_{\mathrm{T}}$, respectively, where

$$
C_{\mathrm{SS}}=\frac{4 \sqrt{2} \pi \alpha_{S}}{3 m^{2}} \frac{1}{\left(2 \pi \Lambda^{2}\right)^{3 / 2}}
$$

and

$$
C_{\mathrm{T}}=\frac{3 \alpha_{S}}{\sqrt{2} m^{2}} .
$$

The expressions (B19) to (B84) given below involve the following quantities:

$$
E=e^{-\rho^{2} / 2 \Lambda^{2}}
$$

TABLE IV. Values of the integrals (A9)-(A13) arising in the calculation of the normalization factors [Eqs. (A2) - (A8)] and $\alpha$ [Eq. (A14)].

\begin{tabular}{ll}
\hline$N_{1}$ & $0.28984 \times 10^{-1}$ \\
$N_{2}$ & $0.57262 \times 10^{-2}$ \\
$N_{3}$ & $0.21158 \times 10^{-2}$ \\
$N_{4}$ & $0.12013 \times 10^{-2}$ \\
$N_{5}$ & $0.21049 \times 10^{-2}$ \\
\hline \hline
\end{tabular}




$$
\begin{array}{ll}
\left.M=\operatorname{erf} \mid \frac{\rho}{\sqrt{2} \Lambda}\right]-\frac{1}{3} \sqrt{2 / \pi} \frac{\rho^{3}}{\Lambda^{3}}\left[1+3 \frac{\Lambda^{2}}{\rho^{2}}\right] e^{-\rho^{2} / 2 \Lambda^{2}}, \\
S_{1}=\langle F|E| F\rangle, \\
S_{2}=\left\langle F\left|\rho^{2} E\right| F\right\rangle, \\
S_{3}=\left\langle F\left|\lambda^{2} E\right| F\right\rangle, \\
S_{4}=\left\langle F\left|\rho^{4} E\right| F\right\rangle, \\
S_{5}=\left\langle F\left|\rho^{2} \lambda^{2} E\right| F\right\rangle, \\
S_{6}=\left\langle F\left|\lambda^{4} E\right| F\right\rangle, \\
S_{7}=\left\langle F\left|\rho^{2} \lambda^{2} P_{2}(x) E\right| F\right\rangle, \\
T_{1}=\langle F|M \rho| F\rangle, \\
T_{2}=\left\langle F\left|\frac{M}{\rho}\right| F\right\rangle, \\
T_{3}=\left\langle F\left|M \frac{\lambda^{2}}{\rho}\right| F\right\rangle, \\
T_{4}=\left\langle F\left|M \frac{\lambda^{2}}{\rho} P_{2}(x)\right| F\right\rangle, \\
T_{5}=\left\langle F\left|M \frac{\lambda^{2}}{\rho^{3}} P_{2}(x)\right| F\right\rangle, \\
T_{6}=\left\langle F\left|M \frac{\lambda^{4}}{\rho^{3}} P_{2}(x)\right| F\right\rangle .
\end{array}
$$

In Eqs. (B11) and (B15) to (B17), $P_{2}$ is the second-degree Legendre polynomial and

$$
x=\frac{\vec{\rho} \cdot \vec{\lambda}}{\rho \lambda} .
$$

As for the quantities (A9)-(A13), the integrals (B5) to (B17) can be reduced to three-dimensional integrals and computed without the Monte Carlo method.

The nonvanishing diagonal and off-diagonal matrix elements are listed below in terms of $S_{i}(i=1-7)$ and $T_{i}$ $(i=1-6)$. As the operators $V_{\mathrm{SS}}$ and $V_{\mathrm{T}}$ are Hermitian, we refer only to the triangle above the main diagonal.

$N\left(\frac{7}{2}^{+}\right)$subspace: $\left\{\left|\Psi_{1}\right\rangle=\left|{ }^{4} N\left(\underline{70}, 2^{+}\right) \frac{7}{2}^{+}\right\rangle\right\}$:

$\left\langle\Psi_{1}\left|V_{\mathrm{SS}}\right| \Psi_{1}\right\rangle=\frac{\left(N_{20}^{\rho}\right)^{2}}{120}\left(3 S_{4}+3 S_{6}+10 S_{5}-4 S_{7}\right), \quad$ (B19)

$\left\langle\Psi_{1}\left|V_{\mathrm{T}}\right| \Psi_{1}\right\rangle=-\frac{\left(N_{20}^{\rho}\right)^{2}}{630}\left(3 T_{1}+7 T_{3}-T_{4}+3 T_{6}\right)$. (B20)

$\Delta\left(\frac{7}{2}^{+}\right)$subspace: $\left.\left\{\left|\Psi_{1}\right\rangle=\left.\right|^{4} \Delta\left(\underline{56}, 2^{+}\right) \frac{7}{2}^{+}\right\rangle\right\}:$

$\left\langle\Psi_{1}\left|V_{\mathrm{SS}}\right| \Psi_{1}\right\rangle=\frac{\left(N_{20}^{S}\right)^{2}}{5}\left(S_{4}+S_{6}+2 S_{7}\right)$,

(B21)

$\left\langle\Psi_{1}\left|V_{\mathrm{T}}\right| \Psi_{1}\right\rangle=-\frac{4\left(N_{20}^{S}\right)^{2}}{105}\left(T_{1}+2 T_{4}+T_{6}\right)$
$N\left(\frac{5}{2}^{+}\right)$subspace: $\left\{\begin{array}{l}\left|\Psi_{1}\right\rangle=\left|{ }^{2} N\left(\underline{56}, 2^{+}\right) \frac{5}{2}^{+}\right\rangle \\ \left|\Psi_{2}\right\rangle=\left|{ }^{2} N\left(\underline{70}, 2^{+}\right) \frac{5}{2}^{+}\right\rangle \\ \left|\Psi_{3}\right\rangle=\left|{ }^{4} N\left(\underline{70}, 2^{+}\right) \frac{5}{2}^{+}\right\rangle\end{array}\right\}:$
$\left\langle\Psi_{1}\left|V_{\text {SS }}\right| \Psi_{1}\right\rangle=-\frac{\left(N_{20}^{S}\right)^{2}}{5}\left(S_{4}+S_{6}+2 S_{7}\right)$,
$\left\langle\Psi_{1}\left|V_{\text {SS }}\right| \Psi_{2}\right\rangle=-\frac{N_{20}^{S} N_{20}^{\rho}}{5 \sqrt{2}}\left(S_{4}-S_{6}\right)$,
$\left\langle\Psi_{2}\left|V_{\text {SS }}\right| \Psi_{2}\right\rangle=-\frac{\left(N_{20}^{\rho}\right)^{2}}{120}\left(3 S_{4}+3 S_{6}+10 S_{5}-4 S_{7}\right)$,

(B23)

(B24)

(B25)

$\left\langle\Psi_{3}\left|V_{\mathrm{SS}}\right| \Psi_{3}\right\rangle=\frac{\left(N_{20}^{\rho}\right)^{2}}{120}\left(3 S_{4}+3 S_{6}+10 S_{5}-4 S_{7}\right)$,

(B26)

$\left\langle\Psi_{1}\left|V_{\mathrm{T}}\right| \Psi_{3}\right\rangle=-\frac{N_{20}^{S}}{15} \frac{N_{20}^{\rho}}{\sqrt{14}}\left(T_{1}-T_{6}\right)$,

$\left\langle\Psi_{2}\left|V_{\mathrm{T}}\right| \Psi_{3}\right\rangle=\frac{\left(N_{20}^{\rho}\right)^{2}}{180 \sqrt{7}}\left(3 T_{1}-7 T_{3}-11 T_{4}+3 T_{6}\right)$,

$\left\langle\Psi_{3}\left|V_{\mathrm{T}}\right| \Psi_{3}\right\rangle=\frac{\left(N_{20}^{\rho}\right)^{2}}{252}\left(3 T_{1}+7 T_{3}-T_{4}+3 T_{6}\right)$.

$\Delta\left(\frac{5}{2}^{+}\right)$subspace: $\left\{\begin{array}{l}\left.\left|\Psi_{1}\right\rangle=\left.\right|^{4} \Delta\left(\underline{56}, 2^{+}\right) \frac{5}{2}^{+}\right\rangle \\ \left.\left|\Psi_{2}\right\rangle=\left.\right|^{2} \Delta\left(\underline{70}, 2^{+}\right) \frac{5}{2}^{+}\right\rangle\end{array}\right\}:$

$\left\langle\Psi_{1}\left|V_{\mathrm{SS}}\right| \Psi_{1}\right\rangle=\frac{\left(N_{20}^{S}\right)^{2}}{5}\left(S_{4}+S_{6}+2 S_{7}\right)$,

$\left\langle\Psi_{2}\left|V_{\mathrm{SS}}\right| \Psi_{2}\right\rangle=\frac{\left(N_{20}^{\rho}\right)^{2}}{40}\left(S_{4}+S_{6}-10 S_{5}-4 S_{7}\right)$,

$\left\langle\Psi_{1}\left|V_{\mathrm{T}}\right| \Psi_{1}\right\rangle=\frac{2\left(N_{20}^{S}\right)^{2}}{21}\left(T_{1}+2 T_{4}+T_{6}\right)$,

$\left\langle\Psi_{1}\left|V_{\mathrm{T}}\right| \Psi_{2}\right\rangle=-\frac{N_{20}^{S} N_{20}^{\rho}}{15 \sqrt{7}}\left(T_{1}-T_{6}\right)$.

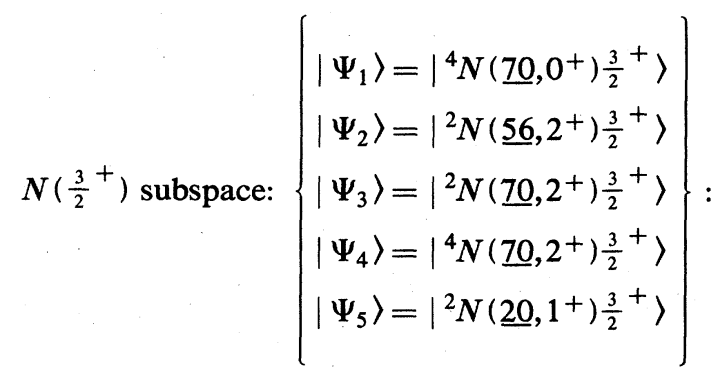

$\left\langle\Psi_{1}\left|V_{\mathrm{SS}}\right| \Psi_{1}\right\rangle=\frac{\left(N_{00}^{\rho}\right)}{96}\left(3 S_{4}+3 S_{6}-2 S_{5}+8 S_{7}\right)$,

(B34)

$\left\langle\Psi_{2}\left|V_{S S}\right| \Psi_{2}\right\rangle=-\frac{\left(N_{20}^{S}\right)^{2}}{5}\left(S_{4}+S_{6}+2 S_{7}\right)$,

(B35) 
$\left\langle\Psi_{2}\left|V_{\mathrm{SS}}\right| \Psi_{3}\right\rangle=-\frac{\sqrt{2}}{10} N_{20}^{S} N_{20}^{\rho}\left(S_{4}-S_{6}\right)$
$\left\langle\Psi_{3}\left|V_{\mathrm{SS}}\right| \Psi_{3}\right\rangle=-\frac{\left(N_{20}^{\rho}\right)^{2}}{120}\left(3 S_{4}+3 S_{6}+10 S_{5}-4 S_{7}\right)$

(B37)

$\left\langle\Psi_{4}\left|V_{\mathrm{SS}}\right| \Psi_{4}\right\rangle=\frac{\left(N_{20}^{\rho}\right)^{2}}{120}\left(3 S_{4}+3 S_{6}+10 S_{5}-4 S_{7}\right)$,

$\left\langle\Psi_{5}\left|V_{\mathrm{SS}}\right| \Psi_{5}\right\rangle=-\frac{2}{9}\left(N_{10}^{A}\right)^{2}\left(S_{5}-S_{7}\right)$,

$\left\langle\Psi_{1}\left|V_{\mathrm{T}}\right| \Psi_{2}\right\rangle=-\frac{N_{00}^{\rho} N_{20}^{S}}{12 \sqrt{5}}\left(T_{1}-T_{3}+T_{4}-T_{6}\right)$,

$\left\langle\Psi_{1}\left|V_{\mathrm{T}}\right| \Psi_{3}\right\rangle=\frac{N_{00}^{\rho} N_{20}^{\rho}}{72 \sqrt{10}}\left(3 T_{1}-7 T_{3}-11 T_{4}+3 T_{6}\right)$,

(B41)

$\left\langle\Psi_{1}\left|V_{\mathrm{T}}\right| \Psi_{4}\right\rangle=\frac{N_{00}^{\rho} N_{20}^{\rho}}{72 \sqrt{5}}\left(3 T_{1}+T_{3}+5 T_{4}+3 T_{6}\right)$,

$\left\langle\Psi_{2}\left|V_{\mathrm{T}}\right| \Psi_{4}\right\rangle=\frac{N_{20}^{\rho} N_{20}^{S}}{30}\left(T_{1}-T_{6}\right)$,

(B42)

$\left\langle\Psi_{3}\left|V_{\mathrm{T}}\right| \Psi_{4}\right\rangle=-\frac{\left(N_{20}^{\rho}\right)^{2}}{180 \sqrt{2}}\left(3 T_{1}-7 T_{3}-11 T_{4}+3 T_{6}\right)$,

$\left\langle\Psi_{4}\left|V_{\mathrm{T}}\right| \Psi_{5}\right\rangle=-\frac{N_{20}^{\rho} N_{10}^{A}}{6 \sqrt{15}}\left(T_{3}-T_{4}\right)$.

(B44)

(B45)

$\Delta\left(\frac{3}{2}^{+}\right)$subspace: $\left\{\begin{array}{l}\left|\Psi_{1}\right\rangle=\left|{ }^{4} \Delta\left(\underline{56}, 0^{+}\right) \frac{3}{2}^{+}\right\rangle \\ \left|\Psi_{2}\right\rangle=\left|{ }^{4} \Delta\left(\underline{56}, 0^{+}\right) \frac{3}{2}^{+}\right\rangle \\ \left|\Psi_{3}\right\rangle=\left|{ }^{4} \Delta\left(\underline{56}, 2^{+}\right) \frac{3}{2}^{+}\right\rangle \\ \left|\Psi_{4}\right\rangle=\left|{ }^{2} \Delta\left(\underline{70}, 2^{+}\right) \frac{3}{2}^{+}\right\rangle\end{array}\right\}:$

$\left\langle\Psi_{1}\left|V_{\mathrm{SS}}\right| \Psi_{1}\right\rangle=\frac{\left(N_{00}^{S}\right)^{2}}{4} S_{1}$

$\left\langle\Psi_{1}\left|V_{\mathrm{SS}}\right| \Psi_{2}\right\rangle=\frac{N_{00}^{S} N_{00}^{S^{\prime}}}{4}\left[S_{1}-\alpha\left(S_{2}+S_{3}\right)\right]$

$\left\langle\Psi_{2}\left|V_{\mathrm{SS}}\right| \Psi_{2}\right\rangle=\frac{\left(N_{00}^{S^{\prime}}\right)^{2}}{4}\left[S_{1}-2 \alpha\left(S_{2}+S_{3}\right)\right.$

$$
\left.+\alpha^{2}\left(S_{4}+2 S_{5}+S_{6}\right)\right]
$$

$\left\langle\Psi_{3}\left|V_{\mathrm{SS}}\right| \Psi_{3}\right\rangle=\frac{\left(N_{20}^{S}\right)^{2}}{5}\left(S_{4}+S_{6}+2 S_{7}\right)$,

(B49)

$\left\langle\Psi_{4}\left|V_{\mathrm{SS}}\right| \Psi_{4}\right\rangle=\frac{\left(N_{20}^{\rho}\right)^{2}}{40}\left(S_{4}+S_{6}-10 S_{5}-4 S_{7}\right)$,

$\left\langle\Psi_{1}\left|V_{\mathrm{T}}\right| \Psi_{3}\right\rangle=\frac{N_{00}^{S} N_{20}^{S}}{3 \sqrt{5}}\left(T_{2}+T_{5}\right)$,

(B38)

(B39)

(B46)

(B47)

(B50)

(B51)
$\left\langle\Psi_{1}\left|V_{\mathrm{T}}\right| \Psi_{4}\right\rangle=-\frac{N_{20}^{\rho} N_{00}^{S}}{6 \sqrt{10}}\left(T_{2}-T_{5}\right)$,

$\left\langle\Psi_{2}\left|V_{\mathrm{T}}\right| \Psi_{3}\right\rangle=\frac{N_{20}^{S} N_{00}^{S^{\prime}}}{3 \sqrt{5}}\left[T_{2}+T_{5}\right.$

$$
\left.-\alpha\left(T_{1}+T_{3}+T_{4}+T_{6}\right)\right]
$$

$\left\langle\Psi_{2}\left|V_{\mathrm{T}}\right| \Psi_{4}\right\rangle=-\frac{N_{20}^{\rho} N_{00}^{S^{\prime}}}{6 \sqrt{10}}\left[T_{2}-T_{5}\right.$

$$
\left.-\alpha\left(T_{1}+T_{3}-T_{4}-T_{6}\right)\right],
$$

(B54)

$\left\langle\Psi_{3}\left|V_{\mathrm{T}}\right| \Psi_{4}\right\rangle=\frac{N_{20}^{\rho} N_{20}^{S}}{15 \sqrt{2}}\left(T_{1}-T_{6}\right)$

(B55)

$N\left(\frac{1}{2}^{+}\right)$subspace: $\left\{\begin{array}{l}\left|\Psi_{1}\right\rangle=\left|{ }^{2} N\left(\underline{56}, 0^{+}\right) \frac{1}{2}^{+}\right\rangle \\ \left.\left|\Psi_{2}\right\rangle=\left.\right|^{2} N\left(\underline{56}^{\prime}, 0^{+}\right) \frac{1}{2}^{+}\right\rangle \\ \left.\left|\Psi_{3}\right\rangle=\left.\right|^{2} N\left(\underline{70}, 0^{+}\right) \frac{1}{2}^{+}\right\rangle \\ \left|\Psi_{4}\right\rangle=\left|{ }^{4} N\left(\underline{70}, 2^{+}\right) \frac{1}{2}^{+}\right\rangle \\ \left|\Psi_{5}\right\rangle=\left|{ }^{2} N\left(\underline{20}, 1^{+}\right) \frac{1}{2}^{+}\right\rangle\end{array}\right\}$.

$\left\langle\Psi_{1}\left|V_{\mathrm{SS}}\right| \Psi_{1}\right\rangle=-\frac{\left(N_{00}^{S}\right)^{2}}{4} S_{1}$,

(B56)

$\left\langle\Psi_{1}\left|V_{\mathrm{SS}}\right| \Psi_{2}\right\rangle=-\frac{N_{00}^{S} N_{00}^{S^{\prime}}}{4}\left[S_{1}-\alpha\left(S_{2}+S_{3}\right)\right]$,

(B57)

$\left\langle\Psi_{1}\left|V_{\mathrm{SS}}\right| \Psi_{3}\right\rangle=-\frac{N_{00}^{S} N_{00}^{\rho}}{4 \sqrt{2}}\left(S_{2}-S_{3}\right)$,

(B58)

$\left\langle\Psi_{2}\left|V_{\mathrm{SS}}\right| \Psi_{2}\right\rangle=-\frac{\left(N_{00}^{S^{\prime}}\right)^{2}}{4}\left[S_{1}-2 \alpha\left(S_{2}+S_{3}\right)\right.$

$\left.+\alpha^{2}\left(S_{4}+2 S_{5}+S_{6}\right)\right]$,

(B59)

$\left\langle\Psi_{2}\left|V_{\mathrm{SS}}\right| \Psi_{3}\right\rangle=-\frac{N_{00}^{S^{\prime}} N_{00}^{\rho}}{4 \sqrt{2}}\left[S_{2}-S_{3}-\alpha\left(S_{4}-S_{6}\right)\right]$,

$\left\langle\Psi_{3}\left|V_{\mathrm{SS}}\right| \Psi_{3}\right\rangle=-\frac{\left(N_{00}^{\rho}\right)^{2}}{96}\left(3 S_{4}+3 S_{6}-2 S_{5}+8 S_{7}\right)$,

(B60)

(B61)

$\left\langle\Psi_{4}\left|V_{\mathrm{SS}}\right| \Psi_{4}\right\rangle=\frac{\left(N_{20}^{\rho}\right)^{2}}{120}\left(3 S_{4}+3 S_{6}+10 S_{5}-4 S_{7}\right)$,

(B62)

$\left\langle\Psi_{5}\left|V_{\mathrm{SS}}\right| \Psi_{5}\right\rangle=-\frac{2\left(N_{10}^{A}\right)^{2}}{9}\left(S_{5}-S_{7}\right)$,

(B63)

(B64) 


$$
\begin{aligned}
\left\langle\Psi_{2}\left|V_{\mathrm{T}}\right| \Psi_{4}\right\rangle= & \frac{N_{00}^{S^{\prime}} N_{20}^{\rho}}{6 \sqrt{10}} \\
& \times\left[T_{2}-T_{5}-\alpha\left(T_{1}+T_{3}-T_{4}-T_{6}\right)\right], \\
\left\langle\Psi_{3}\left|V_{\mathrm{T}}\right| \Psi_{4}\right\rangle= & -\frac{N_{00}^{\rho} N_{20}^{\rho}}{72 \sqrt{5}}\left(3 T_{1}-7 T_{3}-11 T_{4}+3 T_{6}\right), \\
\left\langle\Psi_{4}\left|V_{\mathrm{T}}\right| \Psi_{4}\right\rangle= & -\frac{\left(N_{20}^{\rho}\right)^{2}}{180}\left(3 T_{1}+7 T_{3}-T_{4}+3 T_{6}\right), \\
\left\langle\Psi_{4}\left|V_{\mathrm{T}}\right| \Psi_{5}\right\rangle= & \frac{N_{10}^{A} N_{20}^{\rho}}{3 \sqrt{30}}\left(T_{3}-T_{4}\right) . \\
\Delta\left(\frac{1}{2}^{+}\right) \text {subspace: } & \left\{\begin{array}{l}
\left.\left|\Psi_{1}\right\rangle=\left.\right|^{2} \Delta\left(\underline{70}, 0^{+}\right) \frac{1}{2}{ }^{+}\right\rangle \\
\left.\left|\Psi_{2}\right\rangle=\left|{ }^{4} \Delta\left(\underline{56}, 2^{+}\right) \frac{1}{2}{ }^{+}\right\rangle\right\}
\end{array}\right\} \\
\left\langle\Psi_{1}\left|V_{\mathrm{SS}}\right| \Psi_{1}\right\rangle= & \frac{\left(N_{00}^{\rho}\right)^{2}}{32}\left(S_{4}+S_{6}-6 S_{5}-8 S_{7}\right), \\
\left\langle\Psi_{2}\left|V_{\mathrm{SS}}\right| \Psi_{2}\right\rangle= & \frac{\left(N_{20}^{S}\right)^{2}}{5}\left(S_{4}+S_{6}+2 S_{7}\right), \\
\left\langle\Psi_{1}\left|V_{\mathrm{T}}\right| \Psi_{2}\right\rangle= & \frac{N_{00}^{\rho} N_{20}^{S}}{6 \sqrt{5}}\left(T_{1}-T_{3}+T_{4}-T_{6}\right), \\
\left\langle V_{\mathrm{T}} \mid \Psi_{2}\right\rangle= & -\frac{2\left(N_{20}^{S}\right)^{2}}{15}\left(T_{1}+2 T_{4}+T_{6}\right),
\end{aligned}
$$$$
\left\langle\Psi_{1}\left|V_{\mathrm{T}}\right| \Psi_{1}\right\rangle=-\frac{\left(N_{10}^{\rho}\right)^{2}}{180}\left(T_{2}+T_{5}\right) .
$$

(B66)$$
N\left(\frac{3}{2}^{-}\right) \text {subspace: }\left\{\begin{array}{l}
\left|\Psi_{1}\right\rangle=\left|{ }^{2} N\left(\underline{70}, 1^{-}\right) \frac{3}{2}^{-}\right\rangle \\
\left|\Psi_{2}\right\rangle=\left|{ }^{4} N\left(\underline{70}, 1^{-}\right) \frac{3}{2}^{-}\right\rangle
\end{array}\right\}:
$$

$$
\begin{aligned}
& \left\langle\Psi_{1}\left|V_{\mathrm{SS}}\right| \Psi_{1}\right\rangle=-\frac{\left(N_{10}^{\rho}\right)^{2}}{24}\left(S_{2}+S_{3}\right), \\
& \left\langle\Psi_{2}\left|V_{\mathrm{SS}}\right| \Psi_{2}\right\rangle=\frac{\left(N_{10}^{\rho}\right)^{2}}{24}\left(S_{2}+S_{3}\right), \\
& \left\langle\Psi_{1}\left|V_{\mathrm{T}}\right| \Psi_{2}\right\rangle=-\frac{\left(N_{10}^{\rho}\right)^{2}}{36 \sqrt{10}}\left(T_{2}-T_{5}\right), \\
& \left\langle\Psi_{2}\left|V_{\mathrm{T}}\right| \Psi_{2}\right\rangle=\frac{\left(N_{10}^{\rho}\right)^{2}}{45}\left(T_{2}+T_{5}\right),
\end{aligned}
$$
$\Delta\left(\frac{3}{2}^{-}\right)$subspace: $\left.\left\{\left|\Psi_{1}\right\rangle=\left.\right|^{2} \Delta\left(\underline{70}, 1^{-}\right) \frac{3}{2}^{-}\right\rangle\right\}$.

$$
\left\langle\Psi_{1}\left|V_{\mathrm{SS}}\right| \Psi_{1}\right\rangle=-\frac{\left(N_{10}^{\rho}\right)^{2}}{24}\left(3 S_{2}-S_{3}\right) .
$$

\begin{tabular}{|c|c|c|c|c|c|c|}
\hline$\underset{(\mathrm{fm})}{\Lambda}$ & 0 & 0.065 & 0.130 & 0.195 & 0.260 & 0.325 \\
\hline$S_{1}$ & 0 & $0.56419 \times 10^{-3}$ & $0.31825 \times 10^{-2}$ & $0.71005 \times 10^{-2}$ & $0.11130 \times 10^{-1}$ & $0.14495 \times 10^{-1}$ \\
\hline$S_{2}$ & 0 & $0.64842 \times 10^{-5}$ & $0.11866 \times 10^{-3}$ & $0.46775 \times 10^{-3}$ & $0.10134 \times 10^{-2}$ & $0.16220 \times 10^{-2}$ \\
\hline$S_{3}$ & 0 & $0.10258 \times 10^{-3}$ & $0.58648 \times 10^{-3}$ & $0.13261 \times 10^{-2}$ & $0.20921 \times 10^{-2}$ & $0.27643 \times 10^{-2}$ \\
\hline$S_{4}$ & 0 & $0.12460 \times 10^{-6}$ & $0.75055 \times 10^{-5}$ & $0.53245 \times 10^{-4}$ & $0.16268 \times 10^{-3}$ & $0.32539 \times 10^{-3}$ \\
\hline$S_{5}$ & 0 & $0.11839 \times 10^{-5}$ & $0.22149 \times 10^{-4}$ & $0.89315 \times 10^{-4}$ & $0.19732 \times 10^{-3}$ & $0.32243 \times 10^{-3}$ \\
\hline$S_{6}$ & 0 & $0.35871 \times 10^{-4}$ & $0.20664 \times 10^{-3}$ & $0.47106 \times 10^{-3}$ & $0.74851 \times 10^{-3}$ & $0.99473 \times 10^{-3}$ \\
\hline$S_{7}$ & 0 & $0.93819 \times 10^{-8}$ & $0.40874 \times 10^{-6}$ & $0.25316 \times 10^{-5}$ & $0.71761 \times 10^{-5}$ & $0.13695 \times 10^{-4}$ \\
\hline$T_{1}$ & $0.11674 \times 10^{-1}$ & $0.11452 \times 10^{-1}$ & $0.99072 \times 10^{-2}$ & $0.71062 \times 10^{-2}$ & $0.46389 \times 10^{-2}$ & $0.28926 \times 10^{-2}$ \\
\hline$T_{2}$ & $0.96018 \times 10^{-1}$ & $0.78502 \times 10^{-1}$ & $0.49133 \times 10^{-1}$ & $0.27331 \times 10^{-1}$ & $0.14718 \times 10^{-1}$ & $0.80054 \times 10^{-2}$ \\
\hline$T_{3}$ & $0.18260 \times 10^{-1}$ & $0.15176 \times 10^{-1}$ & $0.97120 \times 10^{-2}$ & $0.55301 \times 10^{-2}$ & $0.30384 \times 10^{-2}$ & $0.16788 \times 10^{-2}$ \\
\hline$T_{4}$ & $0.52082 \times 10^{-3}$ & $0.50257 \times 10^{-3}$ & $0.41005 \times 10^{-3}$ & $0.28332 \times 10^{-3}$ & $0.17806 \times 10^{-3}$ & $0.10783 \times 10^{-3}$ \\
\hline$T_{5}$ & $0.62308 \times 10^{-2}$ & $0.41376 \times 10^{-2}$ & $0.22986 \times 10^{-2}$ & $0.11907 \times 10^{-2}$ & $0.61266 \times 10^{-3}$ & $0.32317 \times 10^{-3}$ \\
\hline$T_{6}$ & $0.21835 \times 10^{-2}$ & $0.14651 \times 10^{-2}$ & $0.83222 \times 10^{-3}$ & $0.44169 \times 10^{-3}$ & $0.23213 \times 10^{-3}$ & $0.12454 \times 10^{-3}$ \\
\hline
\end{tabular}

(B69)

(B70)

Negative-parity states:

$N\left(\frac{5}{2}^{-}\right)$subspace: $\left\{\left|\Psi_{1}\right\rangle=\left|{ }^{4} N\left(\underline{70}, 1^{-}\right) \frac{5}{2}^{-}\right\rangle\right\}$:

$$
\left\langle\Psi_{1}\left|V_{\mathrm{SS}}\right| \Psi_{1}\right\rangle=\frac{\left(N_{10}^{\rho}\right)^{2}}{24}\left(S_{2}+S_{3}\right),
$$

$$
N\left(\frac{1}{2}^{-}\right) \text {subspace: }\left\{\begin{array}{l}
\left|\Psi_{1}\right\rangle=\left|{ }^{2} N\left(\underline{70}, 1^{-}\right) \frac{1}{2}^{-}\right\rangle \\
\left|\Psi_{2}\right\rangle=\left|{ }^{4} N\left(\underline{70}, 1^{-}\right) \frac{1}{2}^{-}\right\rangle
\end{array}\right\}:
$$

(B80)

$$
\begin{aligned}
& \left\langle\Psi_{1}\left|V_{\mathrm{SS}}\right| \Psi_{1}\right\rangle=-\frac{\left(N_{10}^{\rho}\right)^{2}}{24}\left(S_{2}+S_{3}\right), \\
& \left\langle\Psi_{2}\left|V_{\mathrm{SS}}\right| \Psi_{2}\right\rangle=\frac{\left(N_{10}^{\rho}\right)^{2}}{24}\left(S_{2}+S_{3}\right), \\
& \left\langle\Psi_{1}\left|V_{\mathrm{T}}\right| \Psi_{2}\right\rangle=\frac{\left(N_{10}^{\rho}\right)^{2}}{36}\left(T_{2}-T_{5}\right), \\
& \left\langle\Psi_{2}\left|V_{\mathrm{T}}\right| \Psi_{2}\right\rangle=-\frac{\left(N_{10}^{\rho}\right)^{2}}{36}\left(T_{2}+T_{5}\right) .
\end{aligned}
$$

TABLE V. Values of the integrals (B5)-(B17) arising in the calculation of the spin-spin and tensor matrix elements [Eqs. (B19)-(B84)]. 


$$
\left\langle\Psi_{1}\left|V_{\text {SS }}\right| \Psi_{1}\right\rangle=-\frac{\left(N_{10}^{\rho}\right)^{2}}{24}\left(3 S_{2}-S_{3}\right)
$$

We have therefore expressed the 66 nonvanishing matrix elements ( 54 for $\pi=+1$ and 12 for $\pi=-1$ ) of the spin-spin and tensor interactions as simple linear combinations of 13 three-dimensional integrals. The values taken by $S_{i}(i=1-7)$ and $T_{i}(i=1-6)$ as functions of $\Lambda$ are given in Table $\mathrm{V}$.

Note that the vanishing of $S_{i}(i=1-7)$ for $\Lambda=0$ compensates the singularity which appears in the $C_{\mathrm{SS}}$ constant [Eq. (B1)]. Explicitly, one has

$$
\lim _{\Lambda \rightarrow 0} \frac{1}{\left(2 \pi \Lambda^{2}\right)^{3 / 2}} S_{i}=0
$$

for $i=2,4,5$, and 7 while

$$
\begin{aligned}
& \lim _{\Lambda \rightarrow 0} \frac{1}{\left(2 \pi \Lambda^{2}\right)^{3 / 2}} S_{1}=0.15506, \\
& \lim _{\Lambda \rightarrow 0} \frac{1}{\left(2 \pi \Lambda^{2}\right)^{3 / 2}} S_{3}=0.28013 \times 10^{-1}, \\
& \lim _{\Lambda \rightarrow 0} \frac{1}{\left(2 \pi \Lambda^{2}\right)^{3 / 2}} S_{6}=0.97621 \times 10^{-2} .
\end{aligned}
$$

Actually in the limit $\Lambda \rightarrow 0$, the quantities on the left-hand side of Eqs. (B86)-(B88) become one-dimensional integrals.
1J. Carlson, J. Kogut, and V. R. Pandharipande, Phys. Rev. D 27, 233 (1983).

${ }^{2}$ A. De Rújula, H. Georgi, and S. L. Glashow, Phys. Rev. D 12, 147 (1975).

${ }^{3}$ N. Isgur and G. Karl, Phys. Rev. D 18, 4187 (1978).

${ }^{4}$ N. Isgur and G. Karl, Phys. Rev. D 19, 2653 (1979).

${ }^{5}$ N. Isgur and G. Karl, Phys. Rev. D 20, 1191 (1979).

6J. Carlson, J. Kogut, and V. R. Pandharipande, Phys. Rev. D 28, 2807 (1983).
${ }^{7}$ Particle Data Group, Phys. Lett. 111B, 1 (1982).

${ }^{8}$ A. J. G. Hey, P. J. Litchfield, and R. J. Cashmore, Nucl. Phys. B95, 516 (1975).

${ }^{9}$ L. J. Reinders, H. R. Rubinstein, and S. Yazaki, Phys. Lett. B120, 209 (1983).

${ }^{10}$ R. Peierls, Surprises in Theoretical Physics (Princeton University Press, Princeton, New Jersey 1979), p. 61.

${ }^{11}$ R. Koniuk and N. Isgur, Phys. Rev. D 21, 1868 (1980). 